\title{
A retinal circuit that vetoes optokinetic responses to fast
}

\section{visual motion}

Adam Mani, Xinzhu Yang, Tiffany Zhao, David M. Berson

Department of Neuroscience, Brown University, Providence, RI, USA.

\begin{abstract}
Optokinetic nystagmus (OKN) is a visuomotor reflex that works in tandem with the vestibulo-ocular reflex (VOR) to stabilize the retinal image during selfmotion. OKN requires information about both the direction and speed of retinal image motion. Both components are computed within the retina because they are already encoded in the spike trains of the specific class of retinal output neurons that drives OKN - the ON direction-selective ganglion cells (ON DSGCs). The synaptic circuits that shape the directional tuning of ON DSGCs, anchored by starburst amacrine cells, are largely established. By contrast, little is known about the cells and circuits that account for the slow speed preference of ON DSGCs and, thus, of OKN that they drive. A recent study in rabbit retina implicates feedforward glycinergic inhibition as the key suppressor of ON DSGC responses to fast motion. Here, we used serial-section electron microscopy, patch recording, pharmacology, and optogenetic and chemogenetic manipulations to probe this circuit in mouse retina. We confirm a central role for feedforward glycinergic inhibition onto ON DSGCs and identify a surprising primary source for this inhibition - the VGluT3 amacrine cell (VG3 cell). VG3 cells are retinal interneurons that release both glycine and glutamate, exciting
\end{abstract}


some neurons and inhibiting others. Their role in suppressing the response of ON DSGCs to rapid global motion is surprising. VG3 cells had been thought to provide glutamatergic excitation to ON-DSGCs, not glycinergic inhibition, and because they have strong receptive fields surrounds which might have been expected to render them unresponsive to global motion. In fact, VG3 cells are robustly activated by the sorts of fast global motion that suppress ON DSGCs and weaken optokinetic responses as revealed by dendritic $\mathrm{Ca}^{+2}$ imaging, since surround suppression is less prominent when probed with moving gratings than with spots. VG3 cells excite many ganglion cell types through their release of glutatmate. We confirmed that for one such type, the ON-OFF DSGCs, VG3 cells enhance the response to fast motion in these cells, just as they suppress it in ON DSGCs. Together, our results assign a novel function to VGluT3 cells in shaping the velocity range over which retinal slip drives compensatory image stabilizing eye movements. In addition, fast speed motion signal from VGluT3 cells is used by ON-OFF DSGCs to extend the speed range over which they respond, and might be used to shape the speed tuning or temporal bandwidth of the responses of other RGCs.

\section{Introduction}

Imaging-forming visual systems that move through the world, whether biological or artificial, work better when supported by sensorimotor systems for image stabilization. Stabilizing the image on the photoreceptor (or other detector) array simplifies crucial visual computations, such as those needed to distinguish object motion from other sources of visual motion such as eye movements or a 
moving viewpoint). Image-stabilizing reflexes are extremely widespread among animals with image-forming eyes, typically involving multiple sensory drivers and generating compensatory eye, head and/or body movements. In vertebrates, head rotation triggers complementary eye rotation through both vestibular and visual reafference. Slow drift of the retinal image triggers slow compensatory eye movements which eliminate that drift. This visuo-oculomotor reflex is termed optokinetic nystagmus (OKN) [1-3].

To produce image stabilizing eye movements, the OKN works synergistically with the vestibulo-optic reflex (VOR). The VOR is triggered by head movements, which in turn result in inertial forces acting on ampullae of the semicircular canals. When the head undergoes rapid rotation, as during a brisk head movement, the VOR alone nulls most of the resulting retinal slip through counterrotatory eye movements. The remaining retinal slip triggers the OKN which "tops up" the vestibular contribution to eye counterrotation to achieve nearly perfect stabilization. During slow or maintained rotation, OKN plays the dominant role in image stabilization, because biomechanichal constraints limit canal output and thus VOR gain. Therefore, to correctly augment the VOR, the OKR gain is maximal for slower rotational speeds, and as speed gets higher, the augmentation by the OKR is reduced, until it vanishes for sufficiently high speeds. Suppression of the OKR for high speeds is essential for the reflex not to oppose saccades, and specifically those saccades resetting the eye position during the fast phase of OKN.

OKR is mediated by the accessory optic system (AOS), a group of midbrain nuclei that receives direct retinal input. This retinal input is provided by a set of 
direction selective retinal ganglion cells (DSGCs), mainly those of the ON type (ON DSGCs). Retinal ganglion cells (RGCs) are the output neurons of the retina, whose axons transmit all visual information from the retina to the brain. RGCs comprise of $\sim 40$ cell subtypes. RGCs of each subtype pool information from specific presynaptic circuitry, and each convey information about specific aspect(s) of the visual field, such as contrast, direction of motion, orientation, color, or absolute irradiance[4-6].

DSGCs respond best to an image moving in a specific direction. ON DSGCs respond best to slow speeds of retinal slip, typically $\sim 1^{\circ} / \mathrm{s}$, depending on species and stimulus $[2,7,8]$. This is in keeping with their role in driving optokinetic responses. Responses drop markedly for faster speeds of retinal image drift and ON DSGCs spike very little if at all during the rapid rotatory optic flow produced by saccades. By contrast, the ON-OFF DSGCs - a distinct class of direction-selection ganglion cells that contributes to cortical and collicular direction selectivity rather than to image stabilization - respond well to the fast motion that evoke little if any response from ON DSGCs ([9], as confirmed here; One ON-OFF DSGC subtype appears more like ONDS cells in this respect [10]). Among other types of RGCs, responses are sensitive to a large variety of speed ranges, and some are able to respond to very fast motion.

The mechanisms conferring direction selectivity on DSGCs, and specifically ON DSGCs, have been studied in great detail. The retinal mechanisms responsible for the specialized slow speed tuning in ON DSGCs, however, are not yet understood, and mechanisms that shape the motion speed preference of RGCs in general are not well known. 
In principle, the slow speed tuning of ON DSGCs might emerge on several different levels. It may be induced by excitation from presynaptic bipolar cells, depending on their subtype(s) and spatial arrangement of those, as each bipolar subtype has its own characteristic temporal response. Speed tuning can also be determined by inhibition from amacrine cells (ACs), either by contacts onto bipolar cell terminals and reducing excitatory drive, or by feedforward inhibition directly onto the RGC (postsynaptic inhibition). In the latter case, excitatory synaptic currents induced in response to fast motion may be countered by inhibitory currents. Lastly, synaptic currents induced in response to fast motion could be filtered out due to intrinsic electric properties of the RGC.

In a recent study of slow speed tuning of ON DSGCs in rabbit, a postsynaptic, feedforward inhibition has been invoked as its underlying mechanism[8]. A glycinergic inhibitory current has been found to suppress ON DSGC firing in response to a moving full-field stimuli when their speed was high. This inhibitory current was found in ON DSGCs, but not in ON-OFF DSGCs, that are thus able to fire in response to faster motion speeds.

Here we confirm in mouse the earlier findings in rabbit implicating feedforward glycinergic inhibition acting directly on ON DSGC to veto their responses at high speeds. Moreover, we identify the VGluT3 amacrine cell as the primary source for this glycinergic inhibition through convergent evidence from serial block face electron microscopy (SBEM), patch recordings, and cell-type-specific optogenetics and chemogenetics. VGluT3 cells are unusual among amacrine cells because they make excitatory (glutamatergic) as well as inhibitory (glycinergic) synapses onto 
distinct targets [11-13]. Indeed, a prior study found an excitatory glutamatergic VG3 input to ON DSGCs [14]. Though we confirm that in a minority of ON DSGCs, we find that the dominant influence is glycinergic inhibition. We demonstrate that this provides the main basis for vetoing the ON DSGCs response to fast global retinal slip, as during saccades.

Previous studies have shown that VG3 have strong receptive fields surrounds[14,15], which might have been expected to render them unresponsive to global motion. Using functional imaging of calcium responses in the dendrites of VGluT3 ACs, we show that VG3 cells are robustly activated by global motion, and we confirm their high speed preference. These responses evade the surround suppression, which is less prominent when probed with full-field drifting gratings than with bright or dark spots.

Contrary to ON DSGCs, we confirm that the other major class of directionsensitive ganglion cell, ON-OFF DSGCs, receive only excitatory glutamatergic input from VG3 cells. As expected from the fast speed responsiveness of VG3 dendrites and associated suppression of ON-DSGCs, the glutamatergic input to ON-OFF DSGCs augments their responses to fast motion rather than suppressing them. We thus suggest a novel function for VGluT3 cells in sculpting the responses of many RGCs to fast motion, enhancing it in some and inhibiting it in others. In ON DSGCs, it vetoes the response of the image-stabilization system to fast velocities such as those generated by saccades neatly separating these two visuo-oculomotor realms.

\section{Results}


Inhibition at fast speeds underlies slow speed tuning in mouse ON DSGCs.

To explore the mechanisms responsible for speed tuning in ON DSGCs, we made patch recordings of their spiking as well as excitatory and inhibitory currents in whole-mount retinal preparations in response to full-field gratings drifted at a range of speeds. We targeted ON DSGCs by two-photon imaging in mice genetically modified to produce reasonably selective marking by fluorescent reporters (HoxD10GFP or Pcdh9-cre mice; see Methods). In some experiments, we located ON DSGCs in unlabeled retinas by recording extracellular spikes from RGC somas until locating one with characteristic light-evoked responses. The identity of the cells of ON DSGCs was further confirmed by their selectivity for the direction of full-field grating drift and by dye filling and subsequent imaging their characteristic dendritic morphology (see Supplemental Information and Supp. Fig. S1).

As expected, ON DSGC were remarkable among RGCs in preferring slower speeds of grating drifting in the cell's preferred direction (Fig. 1A-C, blue curves). Optimal responses, averaged across cells, were obtained at retinal speeds of $150 \pm 11$ $\mu \mathrm{m} / \mathrm{s}(\mathrm{n}=63)$. This corresponds to an angular velocity of $5^{\circ} / \mathrm{s}$, close to previously reported values for mouse ON-DSGCs and close to the speed producing maximal OKN gain [2]. This value is probably slightly biased upwards because roughly a quarter of the ON-DSGCs tested responded best to the slowest speed tested $(76 \mu \mathrm{m} / \mathrm{s})$ but may have preferred even slower speeds. For speeds above the optimal speed for ON-DSGC firing, the responses sharply decayed, with the spike count decreasing by half at $360 \pm$ $23 \mu \mathrm{m} / \mathrm{s}$. By comparison, diverse other RGC types responded to much higher speeds 
of grating drift (Fig. 1C). ON-OFF DSGCs responded to considerably higher speeds than ON DSGCs (Max. response at $600 \pm 70 \mu \mathrm{m} / \mathrm{s}$, half max. speeds $2000 \pm 160 \mu \mathrm{m} / \mathrm{s}$, ( $n=17)$, Fig. 1C). Some RGCs responded to still higher speeds (e.g. ON alpha RGCs, half max. speed $>3000 \mu \mathrm{m} / \mathrm{s}$ ). An additional RGC type that was slow speed tuned was the ON-delayed RGC[16].

What accounts for the unusual slow speed tuning of ON-DSGCs? Studies in rabbit retina have implicated feedforward glycinergic inhibition of the ganglion cell [8]. So we sought to determine first whether this was true in mice as well, and thus potentially a conserved feature of mammalian retinal image-stabilization networks. To that end, we used whole-cell patch recordings and voltage clamp to measure excitatory and inhibitory currents evoked in ON DSGCs as a function of the speed of retinal slip in the preferred direction (Fig. 1A,B, red, orange curves). Excitatory input was relatively broadly tuned for speed, with maximal excitation observed at about five times the optimal speed for driving spiking (peak amplitude at $710 \pm 90 \mu \mathrm{m} / \mathrm{s}$, (n $=11$ ) or $24^{\circ} / \mathrm{s}$; declining half max. $2300 \pm 170 \mu \mathrm{m} / \mathrm{s}$ ). Excitation was present at the slowest drift speeds we tested, but also at fairly high speeds which evoked no spiking. In sum, though excitatory drive to ON DSGCs exhibits speed tuning, it is clearly insufficient to explain slow speed tuning in ON DSGCs.

Inhibition was small at slow speeds but drew level with excitation at speeds of $\sim 700 \mu \mathrm{m} / \mathrm{s}$ (both normalized by their maxima, Fig. 1A), and always remained high compared to excitation for higher speeds. While the slow speed range was dominated by excitation and spiking, the sharp progressive suppression of spiking occurred over the same range of speeds as a dramatic increase in inhibition. At the highest speeds, 
inhibition remained dominant, suppressing all spiking. Taken together, the above results imply that slow speed tuning of ON DSGCs arises because rapid retinal slip triggers a direct, feedforward inhibition onto the ON DSGC, as has been found in rabbit[8].

For most grating speeds, the excitatory traces had a single prominent peak for each cycle of the grating (Fig. 1B). These peaks likely correspond to a maximal response to positive contrast (ON response), since ON DSGCs are ON dominated. Inhibition traces on the other hand had more 'jagged' shapes, with often two or more local maxima per grating cycle. Since these aligned in time with peaks and troughs in the excitatory current, the inhibitory traces might include a more balanced contribution of the $\mathrm{ON}$ and $\mathrm{OFF}$ pathways of the retina. It is possible that an $\mathrm{ON}-\mathrm{OFF}$ amacrine cell that stratifies within and between the ChAT bands is the source of this inhibition.

As the grating speed increased, the inhibition no longer oscillated between its maximum and zero. Instead, a steady inhibitory current appeared ('DC component'), underneath a continued stimulus-locked modulatory component. The excitatory current also exhibited a much less pronounced DC component. A higher DC shift could be related to a slower (i.e. more sustained) synaptic mechanism, where repeated stimulation (the cycles of the grating) results in non-zero temporal summation. A similar component was observed in ON DSGCs in rabbit[8]. 
To test which neurotransmitter(s) mediate the inhibition at high speeds, we repeated the voltage-clamp measurements in the presence of various synaptic blockers (Fig. 1D,E). Application of strychnine and SR95531 (SR) to block glycine and $\mathrm{GABA}_{\mathrm{A}}$ receptors respectively, removed inhibitory currents in ON DSGCs completely, implying that at least under our stimulation conditions, all feedforward inhibition is mediated by these two type of receptors on the RGC dendrites.

Application of strychnine strongly reduced the inhibitory currents, and the maximum in charge transfer decreased by $57 \pm 4 \%(n=3)$ of its value in the control condition. Strychnine revealed a relatively simple GABA mediated inhibition. The charge transfer depended weakly on speed relative to the control trace, as the peak current increased little with speed, compared to the dramatic increase seen for the control (Fig. 1D). The DC component was strongly attenuated, although not entirely. The trace only had one peak per grating cycle, in contrast to the control. The peaks in current were dramatically out of phase with respect to the peaks in the control condition. This led us to suspect that, in addition to removing a glycine current, the application of strychnine may be introducing GABA currents that are not inherently present (without strychnine). For the slowest speeds this could be seen directly (Fig. 1E, lower left traces). This can occur as a result of blocking of glycine receptors upstream of the RGC dendrites, that releases a GABA signal onto the RGC that would normally be inhibited. If this is indeed the case, then the contribution of glycine to the (control condition) current is larger than would appear from the data in Fig. 1D $(\sim 57 \%)$. 
Application of SR caused a minor change in the currents $(83 \pm 7 \%, n=3)$ and did not alter their speed dependence dramatically. Peaks in the traces seemed slightly more transient relative to the control.

Taken together, these results demonstrate that the inhibition present selectively at high speeds of motion is mediated primarily by glycinergic transmission, as has been found previously in rabbit [8]. 

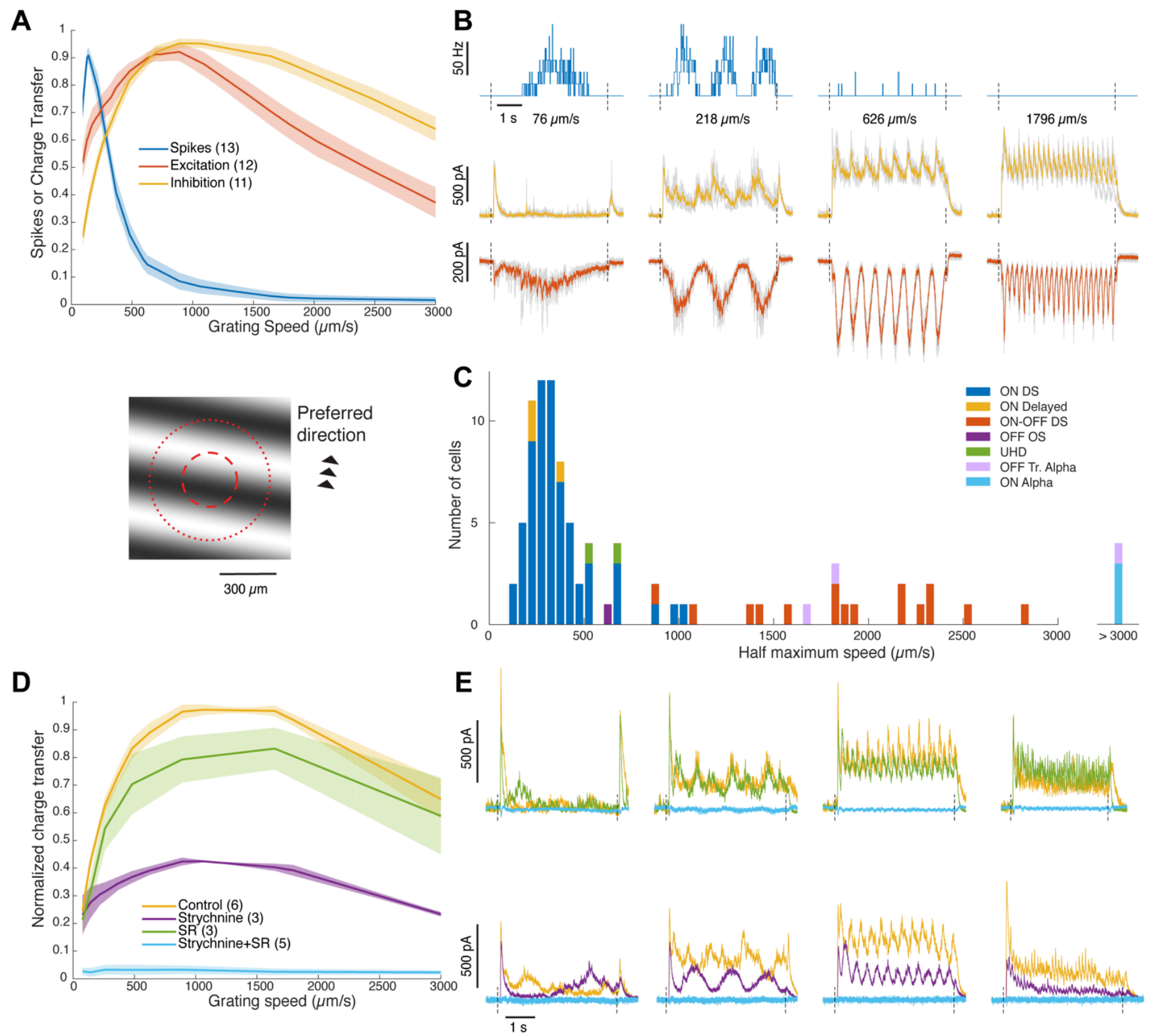

Figure 1. Inhibitory mechanism underlying slow speed tuning in ON DSGCs. (A) Top: Normalized, population averaged speed-response curves of number of spikes (blue), inward charge transfer, membrane voltage clamped to $-65 \mathrm{mV}$ (red), outward charge transfer, voltage clamped to $15 \mathrm{mV}$ (orange) in ON DSGCs. Bottom: gratings stimulus schematics. Red circles: typical center and surround sizes for an ON DSGCs. (B) Spikes, excitatory current $(-65 \mathrm{mV})$, and inhibitory current $(+15 \mathrm{mV})$ responses to different grating speeds in a given ON DSGC. Colors are as in A. (C) Histogram of the declining half maximal response speed (spikes) in RGCs of different types. (D) Inhibitory charge transfer vs. grating speed in ON DSGCs in control conditions (orange), and following application of strychnine (purple), SR95531 (green), or both (light blue). (E) Traces of inhibitory currents for different grating speeds in an ON DSGC, under control conditions, SR95531, SR95531 and strychnine (Top). Or the same in an ON DSGC, but with strychnine applied before SR95531. Colors are as in D. 
Electron microscopic reconstructions identify VGluT3 amacrine cells as the most likely source of glycinergic synapses onto ON DSGCs.

In order to find amacrine cells that are possible sources for the high speed glycinergic inhibition, we examined block face electron microscopy (SBEM) data. In an existing SBEM dataset taken in the inner nuclear layer ('k0725' [17]), we identified dendrites of ON DSGCs. Although a full ON DSGC dendritic tree was too wide to be contained within the retinal area imaged in $\mathrm{k} 0725$, some dendritic trees were complete enough for identification by their dimensions, dendritic morphology and stratification depth within the IPL. Cells identified as ON DSGCs stratified almost entirely in the ON ChAT band, and a large fraction of their amacrine cell inputs originated in ON SACs. The presynaptic SACs formed connections with the ganglion cells in an asymmetric manner, with the connected SAC branches oriented more often in roughly a single direction, as would be expected in a DSGC. Unlike ON-OFF DSGCs the dendrites identified as belonging to ON DSGCs were longer and less dense, and were contained almost entirely in the ON ChAT band, compared to just a few short branches extending into the OFF sublamina of the INL.

The most complete ON DSGC dendritic arbor found in the volume has been identified as an ON DSGC before [18], and its identity has been confirmed here based on the criteria mentioned above (Fig 2A). Along the dendrites of this ON DSGC, we mapped all conventional (i.e., non- ribbon) synapses and then traced the presynaptic cell from each of these identified synapses. We then assigned a cell type with every presynaptic cell whose dendritic tree was complete enough to be identified with a high degree of confidence. Among 448 conventional synapses, 335 belonged to ON 
SACs; 19 belonged to wide-field amacrine cells, characterized by non-branching, long, relatively straight processes; 36 belonged to medium-field amacrine cells that all appear to be VGluT3 amacrine cells. For 58 more synapses, too little information was available to assign a type to the presynaptic cells, mostly because these synapses were close to the boundaries of the imaged volume, and too little of the presynaptic dendritic tree was contained within the volume.

The three dozen medium-field presynaptic amacrine cells, that were identified as VGluT3 ACs (Fig2B, C), cells have been identified by the stratification profiles of their dendrites, specifically by their bushy dendrites stratifying in the layers between the two ChAT bands. The size of their dendrites and coverage factor matched that of VGluT3. In addition, they were all similar in the composition of their presynaptic bipolar cells.

Given the above sample of presynaptic ACs, we asked what cells could provide glycine to support the speed tuning mechanism in ON DSGCs that relies on feedforward glycinergic inhibition. Narrow-field ACs are the main source of glycinergic inhibition in the retina[19], but virtually no cells of this group emerged from the EM analysis. Starburst amacrine cells are well known to be GABAergic (though they also release acetylcholine), and wide-field amacrine cells too have been found to be GABAergic [19-22], with no evidence for glycinergic varieties [20]. This leaves only the VGluT3 cells, a dual neurotransmitter amacrine cell that releases both glycine or glutamate. VGluT3 ACs thus emerged in SBEM data as candidates to be a source for glycine onto ON DSGCs, and possibly the only one, that provides inhibition at high motion speeds. 
A

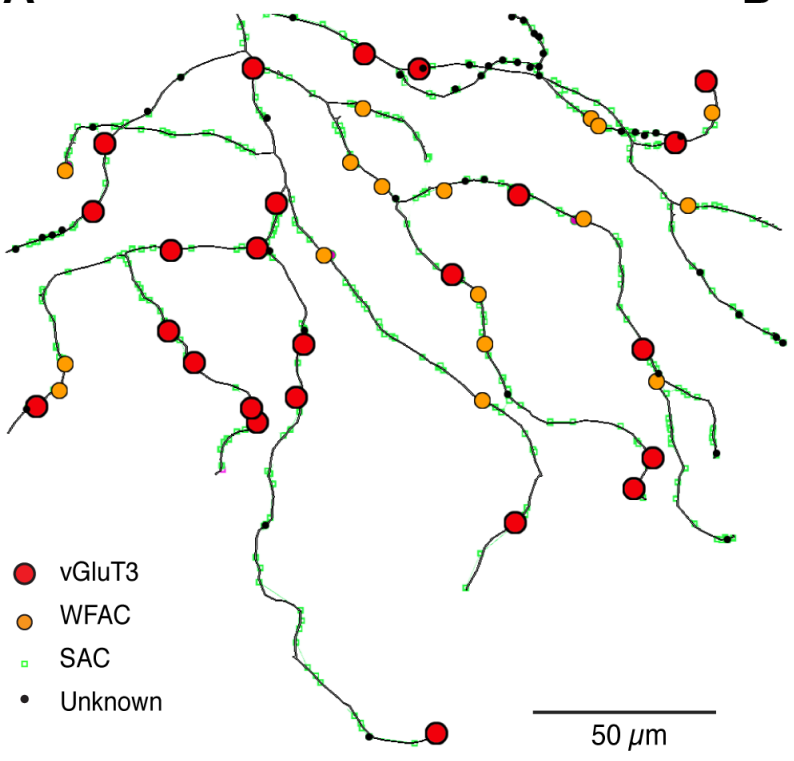

B
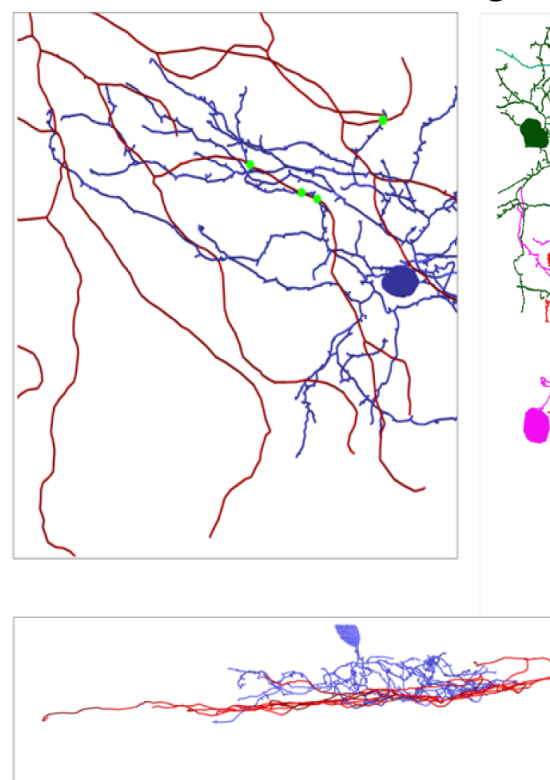

C

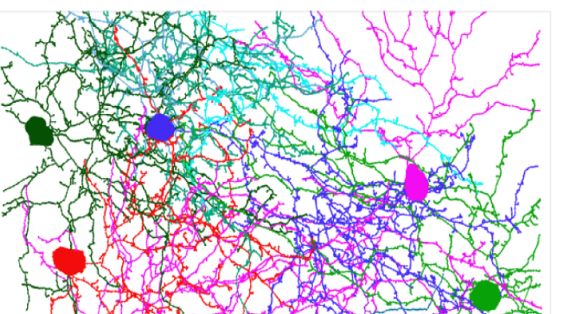

Figure 2. Amacrine cell inputs onto an ON DSGC. (A) Top view (top) and a side view (bottom) of a reconstruction of an ON DSGC within the SBEM dataset volume. Different types of markers labels synapses of VGluT3 ACs, wide-field ACs, SACs and synapses with an unknown presynaptic cell. Gray traces denote SAC processes. (B) Top and side view (top, bottom respectively) of a single VGluT3 cell (purple), the ON DSGC from A (red) and synapses connecting them (light green). (C) All traced cells in the volume identified as VGluT3 ACs. 


\section{VGluT3 cells form functional glycinergic synapses with ON DSGCs}

ON DSGCs have been identified previously as postsynaptic targets of VGluT3. That study, however, found that VG3 cells released glutamate onto ON DSGCs rather than glycine. In order to reexamine whether VGluT3 cells might make functional glycinergic inhibitory connections with ON DSGCs, we depolarized VGluT3 cells optogenetically while recording postsynaptic currents in ON DSGCs. To this end we crossed a novel VGluT3-cre mouse with a mouse expressing channelrhodopsin2 (ChR2) in a cre dependent manner (Ai32) (See Supplemental Information, Supp. Fig. S2). The native phototransduction of the retina, as well as acetylcholine receptors, were blocked using a cocktail of synaptic blockers ([14] 'photoreceptor block' [14], See Methods). In $81 \%$ of ON DSGCs tested (13/16 cells), we recorded inhibitory postsynaptic currents, with a peak current of $33 \pm 4$ pA (Fig. 4A-C).

In order to assess which neurotransmitter(s) mediated the inhibitory current induced optogenetically, we used standard synaptic blockers in addition to the photoreceptor block. In the following order, we added to the bath (1) the photoreceptor block (L-AP4, ACET, Hexamethonium), (2) Glutamate receptor blockers (CNQX, D-AP5), (3) glycine receptors blocker (strychnine). (Fig 4B). Blocking glutamate receptors did not eliminate the optogenetically induced inhibitory current (decrease in maximal current by $28 \pm 7 \%, 5$ cells). The subsequent blocking of glycine receptors removed the current nearly completely (decrease by $81 \pm 5 \%, 4$ cells). The blocking of glutamate receptors prior to blocking glycine receptors further reduced the chance of the current being the result of the retinal native phototransduction and glutamate release from bipolar cells (onto a presynaptic amacrine cell). It also 
eliminated the possibility of an indirect interaction between VGluT3 ACs and the ON DSGC through VGluT3 cells releasing glutamate (see next paragraph). The result above strongly supports the existence of a functional glycinergic synapse from VGluT3 cells onto ON DSGCs.

To further assess the contribution of the glycinergic synapses to the inhibitory current induced by optogenectics in ON DSGCs, we switched the order of steps in the previous experiment, namely, we blocked glycine receptors first, and glutamate receptors later (Fig 4C). Strychnine blocked the inhibition completely in one out of three cells, but not in two others. Glutamate blockers added afterwards blocked the inhibitory current. We suggest that the current not blocked by strychnine results indirectly from actions of optogenetically activated VGluT3 cells on intermediate amacrine cells. In this case VGluT3 may cause excitation of the intermediate amacrine cell by releasing glutamate, while the later induces inhibition in the ON DSGCs by releasing GABA. More data, however, would be needed to properly determine the source of the additional current.

Over the course of a recording and repeated stimulation for optogenetics, a gradual rundown of currents was observed. In order to verify that the elimination of the current is indeed the result of synaptic blockade, rather than rundown, we conducted sham experiments (Fig 4D). Following photoreceptor block in ON DSGCs, we measured the currents several times at time intervals similar to those measured in the pharmacology experiments. The peak current reduced by $38 \pm 2 \%$ ( 2 cells) over two steps, but was not eliminated. Rundown could, however, be the cause of partial decreases of the current between steps in these experiments. A second complication 
were occasional large currents that may have been the result of incomplete blocking of photo-transduction. Those were mostly inconsistent over trials and had a longer latency compared to persistent optogenetic responses, and were excluded from the data.

In control ON DSGCs recorded in animals not carrying the ChR2 transgene, we did not record stimulus locked currents like those presented in the optogenetics data above. In both the data and control, occasional non-stimulus locked bursts were sometimes seen, as well as a persistent inhibitory OFF light response in some ON DSGCs (namely, outside the LED stimulation interval that preceded it), that was resistant to ACET.

In VGluT3 x Ai32, we observed expression of the reporter in Müller Glia. Although an interaction between Müller glia and ON DSGCs in our experiments is unlikely, interactions between Müller glia and amacrine cells have been reported previously[23]. In order to rule out Müller glia as a possible source for the currents we observed, we crossed a mouse expressing cre in Müller Glia (Glast-creER) with the Ai32 mouse. Recording ON DSGCs in these animals, we did not detect any currents (inhibitory or excitatory) in response to the LED light.

As a further test of our optogenetics scheme and the VGluT3 x Ai32 mouse, we recorded optogenetically induced currents in other RGCs. Inhibitory current was recorded in a Suppressed by Contrast RGC (Fig 4E), and an excitatory current in an ON-OFF DSGC (Fig 2I), in agreement with previous work[14,24,25].

In order to reliably suppress spiking in response to fast moving patterns, inhibition from VGluT3 into ON DSGCs has to be robust to rapid changes in contrast. 
We therefore tested if the inhibition induced by optogenetics would survive when VGluT3 ACs are stimulated by a pulse train of LED stimulation (Fig. 4F). Keeping an optimal pulse width of 100 ms to robustly induce current but minimize rundown, we varied the frequency between $1 \mathrm{~Hz}$ and $8 \mathrm{~Hz}$, a range corresponding to most of the temporal frequency range affecting the retina in our experiments using varying grating speed ( $1 \mathrm{~Hz}$ corresponding to a speed of $380 \mu \mathrm{m} / \mathrm{s}$ ). The inhibitory current remained stable for five seconds of stimulation for the entire frequency range. For 8 $\mathrm{Hz}$ frequency, the oscillation is about a steady non-zero component, similar to the inhibition in response to gratings (compare to Fig 1B). Although the nature of the stimulation in this experiment is obviously different than the retina's native light response, it demonstrates robustness of the current response to flicker, at least at the level of the VGluT3-ON DSGC synapse.

These findings support the hypothesis that VGluT3 ACs make glycinergic synapses onto ON DSGCs, as required if they are to constitute the source of the feedforward inhibition that suppresses the responses to ON DSGCs to fast retinal slip.

Previous work found excitatory current from VGluT3 to ON DSGCs induced by optogenetic stimulation of VGluT3[14]. Recording ON DSGCs held at the chloride reversal potential and stimulating with blue light, we observed excitatory currents rarely, with detectable currents in only $12 \%$ of cases (2/16 cells), with peak currents of $10 \pm 1 \mathrm{pA}$. It might be that introducing glycine or GABA receptor blockers without blocking glutamate receptors would reveal excitatory currents more often, or larger currents (for example by removing basal presynaptic inhibition) as we indeed observed in one cell (Fig. 4F, G). In both ON DSGCs where excitation was observed, 
inhibition was observed as well, demonstrating the existence of excitation and inhibition from VGluT3 in the same RGC type, as well as in the same cell. This is in contrast to previous suggestions that VGluT3 is either solely inhibitory or excitatory in given RGC types[25], and is reminiscent of DSGCs receiving both excitatory and inhibitory inputs from starburst amacrine cells in creating directional tuning in DSGCs. 
A

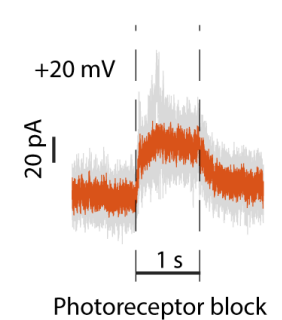

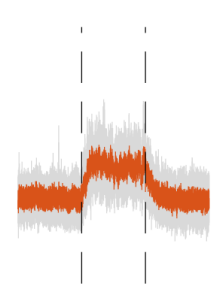

+ CNQX +D-AP5
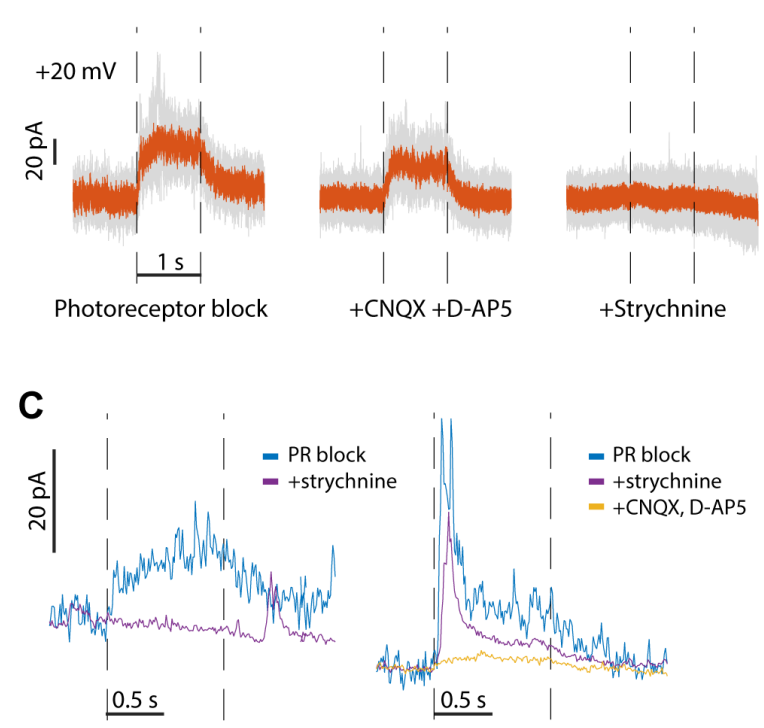

\section{$F$}
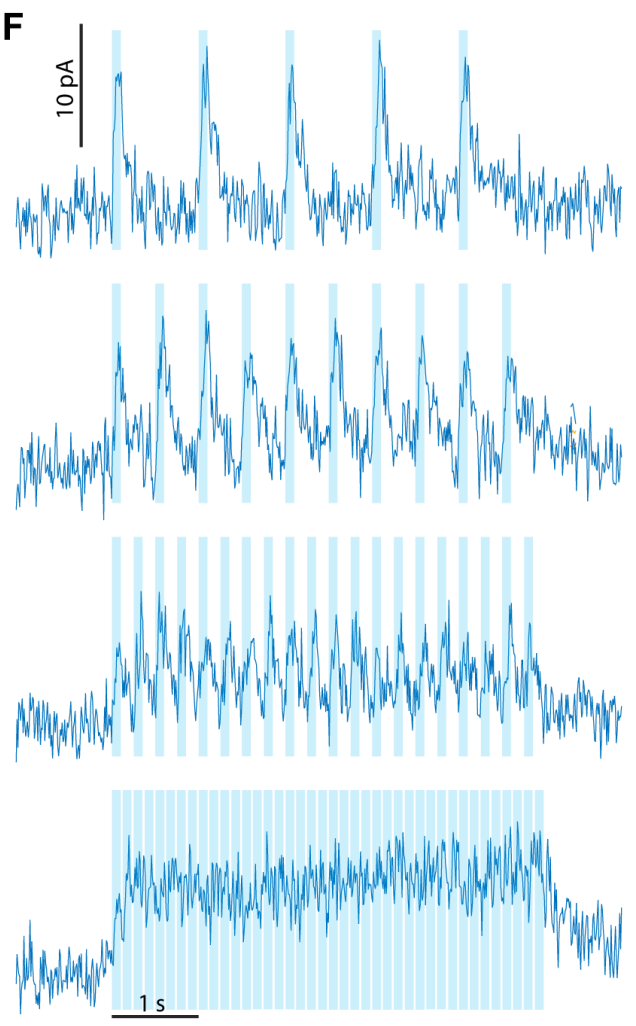

B
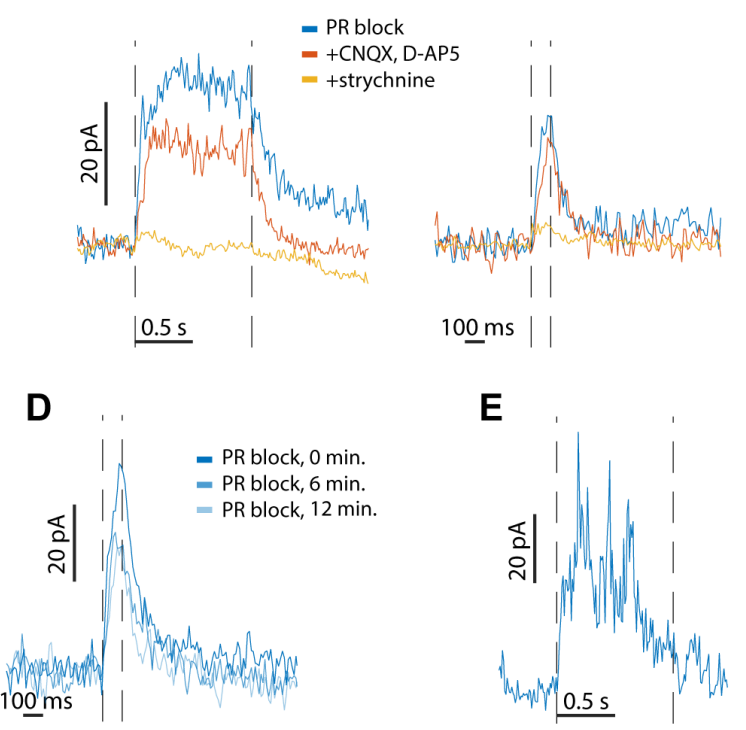

Suppressed by Contrast

G
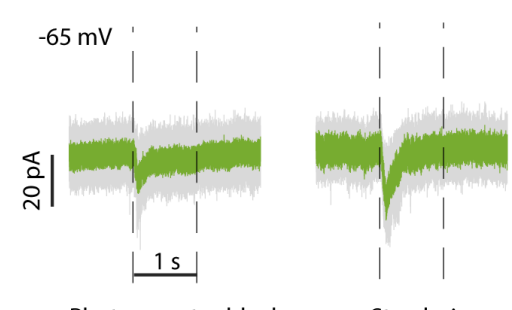

+Strychnine

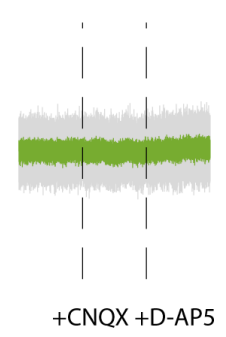

H

I

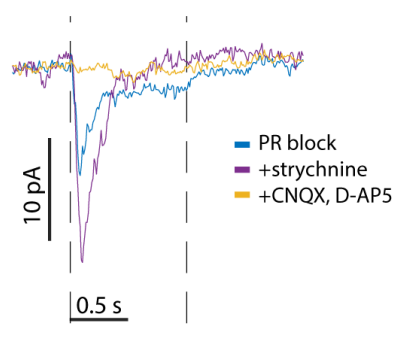

ON-OFF DSGC

Figure 4. Postsynaptics currents in ON DSGCs from optogenetic activation of VGluT3. (A) Raw inhibitory current traces in an ON DSGC, in response to LED light. "Photoreceptor block" is L-AP4, ACET and Hexamethonium. Other synaptic blockers were added in two additional steps. Holding voltage is $+20 \mathrm{mV}$ in A through F. LED light was ON between the dashed lines. (B) Smoothed traces of inhibitory currents in a three step pharmacology experiment, in two cells. Left, same cell as in A. (C) Inhibitory 
currents in a pharmacology experiment in two cells, strychnine applied before CNQX, D-AP5 rather than vice-versa. (D) Sham three step experiment, no blockers apllied. (E) Inhibitory current from optogenetic activation of VGluT3 in a Suppressed by Contrast RGC, in the presence of photoreceptor block. (F) Excitatory currents in an ON DSGCs in response to pulse trains of frquencies 1, 2, 4, $8 \mathrm{~Hz}$. Pulse width is $100 \mathrm{~ms}$. (G) Raw ecitatory current traces in an ON DSGC, in response to LED light. Holding voltage is $-65 \mathrm{mV}$ in $\mathrm{G}$ through $\mathrm{I}$. (H) Pharmacology experiment, excitatory currents in same ON DSGC as in G. (I) Excitatory current from optogenetic activation of VGluT3 in an ON-OFF DSGC, in the presence of photoreceptor block.

VGluT3 ACs contribute a major part to the inhibition onto ON DSGCs at high speeds

In order to test for the existence and role of VGluT3 mediated inhibition at high motion speeds, we had the retina express an inhibitory DREADD (Designer Receptor Exclusively Activated By Designer Drugs) in VGluT3 ACs. The receptor hM4Di, a modified human muscarinic receptor that does not naturally occur in mouse, is activated by binding the ligand Clozapine-N-Oxide (CNO), and causes hyperpolarization in the neurons expressing it, suppressing their neurotransmitter release. We expressed DREADDs in VGluT3 ACs either by crossing VGluT3-cre mice with mice expressing cre-dependent DREADD, or using an adeno-associated virus (AAV) carrying DNA encoding cre-dependent DREADD administered via an intraocular injection to the VGluT3-cre mouse. We then recorded inhibition in ON DSGCs in response to drifting gratings, with and without CNO in the bath medium (Fig.5A).

In the VGluT3-cre mouse injected with DREADD AAV, inhibition recorded in response to gratings (Fig. 5A-i) was similar to that recorded in wild-type animals (Fig.1). Following application of CNO, inhibition dramatically decreased for all speeds, with the maximum decreased by $43 \pm 7 \%(n=3)$ of its control condition peak. The results were similar when the same recordings were done in retinas from the VGluT3- 
DREADD mouse, with inhibition in the presence of CNO decreasing by $38 \pm 6 \%(n=3)$ of the control condition at the peak (Fig. 5A-ii). In a control experiment where CNO was applied while recording inhibition in an ON DSGC in the HoxD10 mouse lacking DREADDs, no similar effect was observed (Fig. 5A-iii).

According to the simple pharmacology experiments, glycine accounted for at least $57 \%$ of the inhibition in the control condition (Fig. 1D). Applying strychnine to the bath following the application of CNO, a further decrease in inhibition occurred, reducing the maximum of the curve by $72.8 \pm 0.5 \%(n=2)$ of the maximum in the control condition (Fig. 5B), thus suggesting that glycine might account for $73 \%$ of the inhibition. This was true regardless of whether the DREADD had been introduced by the AAV or the mouse line crossing. The difference might point to a GABA current onto the ON DSGC from an amacrine cell activated by glutamate release from VGluT3, as suggested in the optogenetics experiments.

The effect of DREADD in blocking high speed inhibition was thus partial $(\sim 40 \%)$. The close similarity in the result in both cases, where DREADDs were introduced by an AAV or through the crossed mouse line, reduces the probability of the partial effect being due to inefficiency of transfection by the AAV. It could be the result of DREADDs only partially hyperpolarizing VGluT3 ACs, or only expressed in some of the VGluT3 ACs. Alternatively there could be another glycine producing cell population that have synapses with ON DSGCs, but this is unlikely due to the SBEM data.

Unlike the effect of strychnine in the standard pharmacology experiments (Fig. 1D), that changed the kinetics of the inhibitory currents, and may have introduced 
currents not present as part of the control condition inhibition (see first section of Results), application of CNO preserved the kinetics of the inhibitory currents. The effect of CNO application was scaling down of the current relative to the control condition. This was evident both in the charge transfer vs. speed curves (fig. 5A, B), as well as in the current traces themselves, especially for higher speeds (Fig. 5C, D). The relative contribution of VGluT3 ACs (The part removed by CNO) as a fraction of the control inhibitory currents, remained relatively constant above speeds of $\sim 600 \mu \mathrm{m} / \mathrm{s}$ (Fig. 5E). For lower speeds the scatter in this factor was larger due to smaller currents, and possibly a contribution from non-VGluT3 sources that would have a larger relative contribution.

Although we could not rule out the contribution of non VGluT3 cells based on the DREADD experiments, the preserved kinetics suggests that the control inhibitory currents at high speeds may be produced mostly or entirely by vglut2 ACs. If the DREADD system is only partially effective in silencing VGluT3 cells (or is only expressed in a fraction of them), this can scale down glycine release by a constant factor, for as long as CNO is present in the bath. In that case, this factor would be independent of time (in the current traces) or the grating speed. The absence of an additional glycniergic population in the SBEM data supports this scenario.

To test whether we could see a direct effect on the speed tuning of an ON DSGC by the presumed reduced activity of VGluT3, we repeated the DREADD experiment, recording the cell's spiking in a cell-attached configuration. The effect, although not large, was in the predicted direction, with the response curve shifted towards higher speed (Fig. 5F). We then added strychnine, that caused a more dramatic shift in the 
curve towards higher speeds, in a similar way to that found in rabbit[8], and in agreement with the larger decrease in inhibition seen with strychnine. The effect of CNO is relatively small possibly due to its partial effect on inhibition, that might be reduced even more at the level of spiking, by spiking nonlinearity in the ON DSGC.

Taken together, the above results suggest that the native inhibition in $\mathrm{ON}$ DSGCs at high speeds is provided at least to a large extent by glycine release from VGluT3 ACs, although our manipulation to suppress VGluT3 signaling was only partially effective.

VGluT3 cells augment responses to higher speeds in ON-OFF DSCGs

VGluT3 ACs have been previously found to provide excitation to ON-OFF DSGCs in optogenetics experiments[14,24], an excitation that we were able to replicate (Fig.4I). Unlike ON DSGCs, ON-OFF DSGCs exquisitely detect motion up to rather high speeds (Fig. 5G, 1C). We asked whether VGluT3 ACs could be a source for a high speed signal for ON-OFF DSGCs as well. Since VGluT3 ACs in this case provide excitation, it would increase the DSGCs' ability to detect high motion speeds rather than suppressing such responses. Recording ON-OFF DSGCs in VGluT3-DREADD mice, we measured excitation in response to drifting gratings of different speeds, in the cells' predetermined preferred direction (Fig. 5H). Comparing excitation before and after application of CNO, excitation was indeed reduced, by $17 \pm 3 \%$ at its maximum charge transfer, relative to the control condition. Unlike the case for ON DSGCs, the curves in this case were not merely scaled down, but were shifted towards lower 
speeds at the falling branch $(\Delta=400 \pm 160 \mu \mathrm{m} / \mathrm{s}$ at half max. of the control curves $)$, with hardly any shift at the rising branch.

The small decrease in excitation (17\%), as well as a shift in the curve rather than mere scaling, are to be expected, since VGluT3 is unlikely to be the sole source of excitation, even at high speeds, due to the strong drive from bipolar cells to the ONOFF DSGC. Those would have, in general, different response kinetics than VGluT3 ACs.

Since for motion in the preferred direction ON-OFF DSGCs spiking closely follow excitation (Fig. 5G), the above result supports the hypothesis of VGluT3 increasing ON-OFF DSGC response at high speeds. In order to try and observe an effect directly on spiking, we repeated the DREADD experiment, recording spikes in an ON-OFF DSGC in response to the gratings (Fig. 5H). Indeed an effect on spiking was observed as well here, with the spike responses vs. speed curve moving slightly towards lower speeds.

The results above show that VGluT3 may be a source of a high speed signal to the entire direction selective (DS) system of the retina, with an opposite effects in $\mathrm{ON}$ OFF DSGC and in ON DSGCs. While suppressing responses by feedforward inhibition at fast speed in ON DSGCs, it enhances responses in ON-OFF DSGCs at high speeds by providing excitation. 

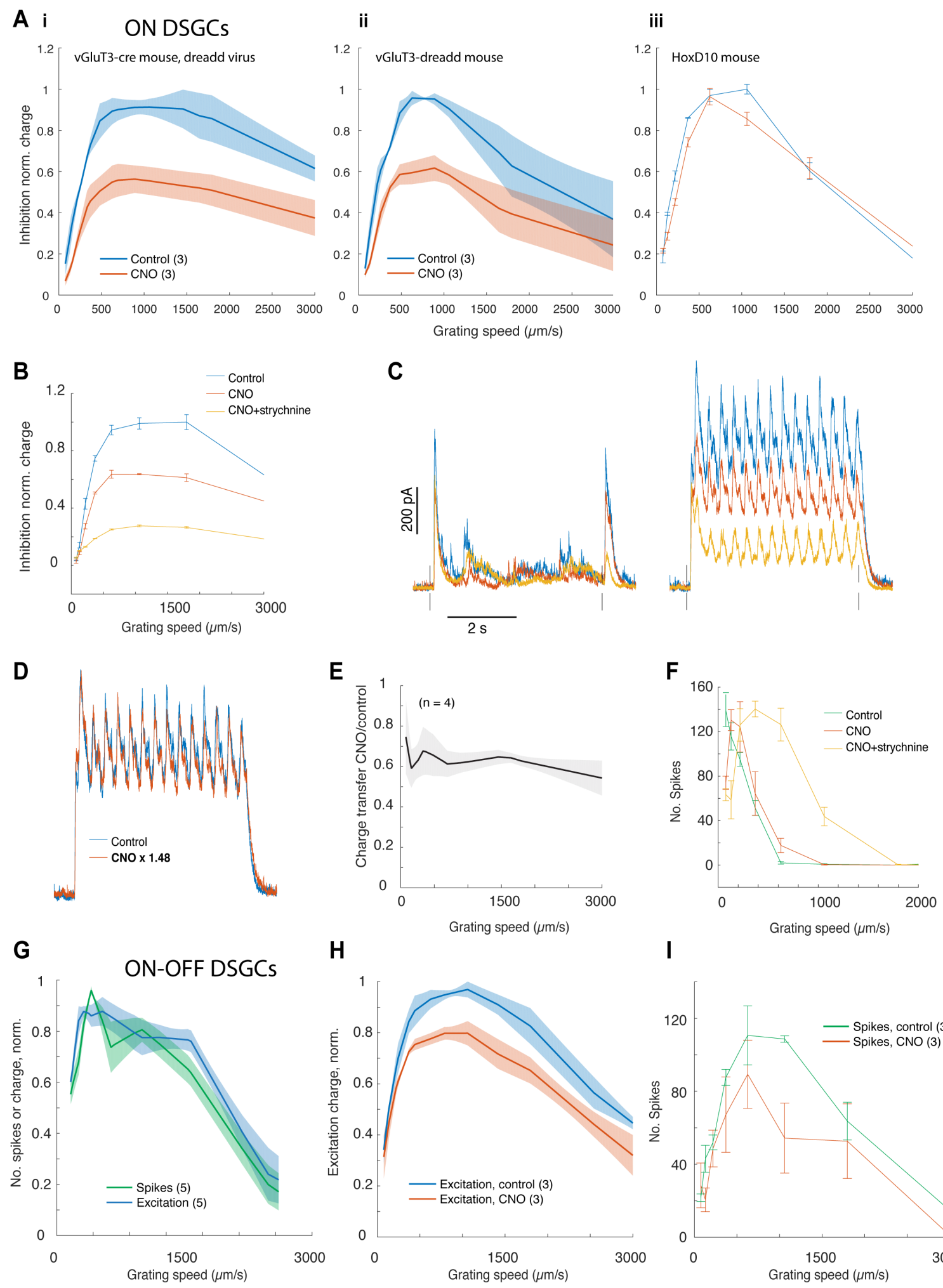

I

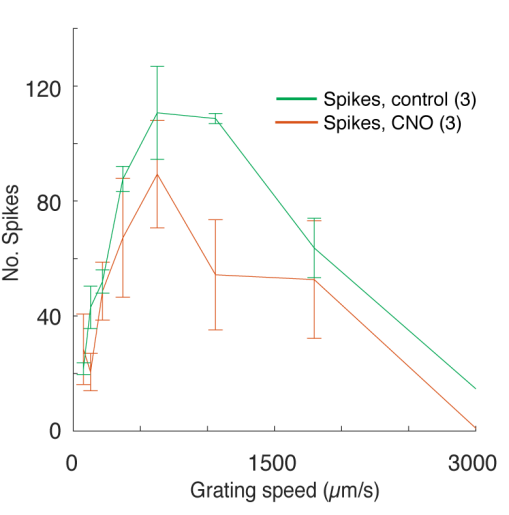

Figure 5. The effect of an inhibitory DREADD in ON and ON-OFF DSGCs. (A) ON DSGC inhibition $(+15 \mathrm{mV})$ vs. grating speed in control conditions (blue) and following CNO application (red) in the VGluT3-cre mouse infected with a DREADD carrying virus (i), in the VGluT3xR26 DREADD mouse (ii) or in a control HoxD10 mouse (iii). (B) ON DSGC inhibition vs. grating speed in a VGluT3-cre 
transfected with a DREADD carrying AAV, in an control conditions, following CNO, CNO+strychnine. (C) Individual current traces of the ON DSGC in B, at a low (left) and high (right) grating speeds. (D) The control and CNO current traces from $\mathrm{C}$, with the CNO trace rescaled by 1.48 to superimpose it on the control trace. (E) The ratio of inhibition with $\mathrm{CNO}$ and in the control condition vs. grating speed, in DREADD carrying VGluT3 mice, averaged over 4 ON DSGCs. (F) Number of spikes vs. grating speed in an ON DSGC in a VGluT3xR26 DREADD mouse, in control conditions, with CNO, and CNO+strychnine. (G) Spiking in ON-OFF DSGCs vs. grating speed along with their excitation (H) Excitation in ON-OFF DSGCs vs. grating speed, VGluT3XR26 DREADD mouse, before and after application of CNO. (I) Spikes in an ON-OFF DSGCs vs. grating speed, VGluT3xR26 DREADD mouse, before and after application of CNO.

\section{Direct recording of VGluT3 AC signaling of high speed motion}

The experiments described above strongly support the role of vGluT3 ACs in signaling high speed motion to ON DSGCs, and possibly other postsynaptic targets, through recordings in the postsynaptic cells. We wanted a direct confirmation of the same responses in VGluT3 ACs themselves. In addition, VGluT3 ACs have been found in past work to possess strong surround suppression[14,15]. This may seem a priori as contradictory to their newly proposed role of signaling full-field fast motion, as responses for a spatially extended stimulus would be strongly suppressed. Spatial receptive field properties such as surround suppression can in general depend on the type of stimulus used to measure them ([26] and refs. therein), and previous measurements relied on area-response curves using static, bright or dark spots or annuli.

We thus turned to calcium imaging in VGluT3 ACs, by crossing the vglut3-cre mouse with a mouse carrying a cre dependent calcium indicator (Ai148). Recording calcium responses in somata of VGluT3 ACs, we hardly detected changes in fluorescence, in response to any light stimulus, possibly due to thin cytosol, or small concentration of calcium channels in the VGluT3 soma. However, examination of the 
plexus of VGluT3 dendrites revealed rich light responses. We acquired time traces of the average relative change in fluorescence in manually labeled ROIs (Fig. 6A).

Robust responses were recorded in response to full-field drifting gratings of different speeds (The grating angle was arbitrary and kept constant throughout the stimulus set). Moreover, the responses were strongly tuned to higher speeds (Fig. 6B, C). Most ROIs in a $120 \mu \mathrm{m}$ x $60 \mu \mathrm{m}$ field of view yielded similar speed response curves. Figure 6C shows average curves over ROIs, for four different fields of view. The shape of the curve possibly depends on the direction of the grating, and on the location on the retina. However further study is needed in order to quantify these effects. We did not observe appreciable dependence of the curve on the depth of the imaged plane within the IPL, which implies that the responses were not limited to the ON or OFF dendrites of VGluT3.

In order to test the effect of surround suppression on the responses, we presented full-field gratings, and the same gratings limited to a circular mask (230 $\mu \mathrm{m}$, centered on the area of recording), in the same field of view (Fig. 6D). Responses were indeed much stronger with the masked grating. The high speed preference was preserved, although the responses started appearing for lower speeds.

To further test the question of surround suppression, we measured arearesponse curves. We used either bright spots of varying diameters as has been done previously [15], or we presented gratings, limited by circular masks of varying diameters (Fig. 6E). Using spots, we observed calcium responses in the dendrites that were in agreement with previous findings, that had been acquired by somatic recording of membrane currents. The responses indeed had a strong surround 
suppression, with peak responses at a spot diameter of $204 \pm 10 \mu \mathrm{m}$ (20 ROIs), and responses down to $8 \pm 1 \%$ of their maximum at the largest diameter tested $(930 \mu \mathrm{m})$ for ON responses, and $217 \pm 12 \mu \mathrm{m}$ (19 ROIs) maximal response diameter, $4 \pm 1 \%$ suppression for OFF responses (bright spot disappearing).

We then repeated the measurement, replacing the static bright spots with masked gratings drifting at a high speed $(1520 \mu \mathrm{m} / \mathrm{s})$, and varying the diameter of the mask. The shape of the resultant receptive field was strikingly different than in the case of spots. Surround suppression was now weaker, as evident by the maximum response that now peaked at much larger diameters $408 \pm 10 \mu \mathrm{m}$ (28 ROIs), and by the responses at the largest diameter tested reduced only to $26+3 \%$ relative to their maximum. The surround suppression strength of VGluT3 ACs is thus stimulus dependent, enabling them to respond to global motion, even though they are strongly suppressed by large spots.

The difference in surround suppression between the two stimuli (Fig. 6E) could arise from the presence or absence of continuous motion. Alternatively it could be the result of the change in contrast. The spot stimulus by its nature is a step in overall contrast (positive or negative) with respect to the preceding and surrounding background. The gratings however, were presented on a gray background, with the mean change in overall contrast being zero. We thus tested new sets of spots and gratings, where the overall contrast in the two stimuli was equal and positive (Fig. 6F; see methods). The receptive field measured with spots had a shape similar to the previous (Fig. 6E) despite a lower contrast, although responses were weaker. The surround suppression for masked gratings, however, was stronger than for zero mean 
gratings. Yet, the receptive field still had a larger spatial extent than that of the receptive field measured with spots of equal contrast (Fig. 6F). Therefore, the weaker surround suppression in the case of gratings vs. spots, is both due to a smaller/no change in contrast, and due to the continuous motion. Under natural conditions of slow drift of the visual scene on the retina, no sudden appearance or large changes in contrast occur, and thus VGluT3 ACs should detect the motion with minimal suppression.

Taken together our results suggest that VGluT3 ACs are fit to report global image slip, as their effective receptive field in this case is large, and does not exhibit a prohibitive surround suppression. 

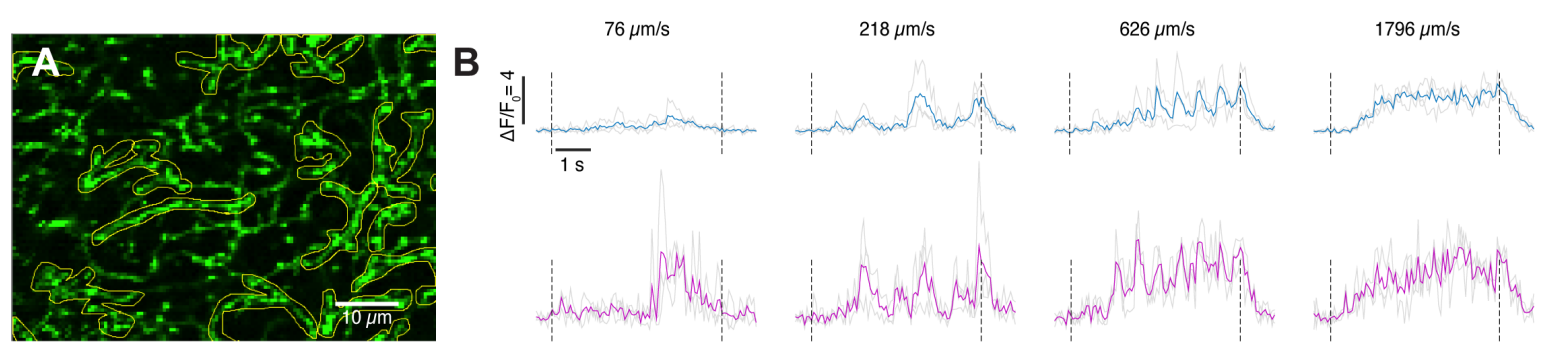

C
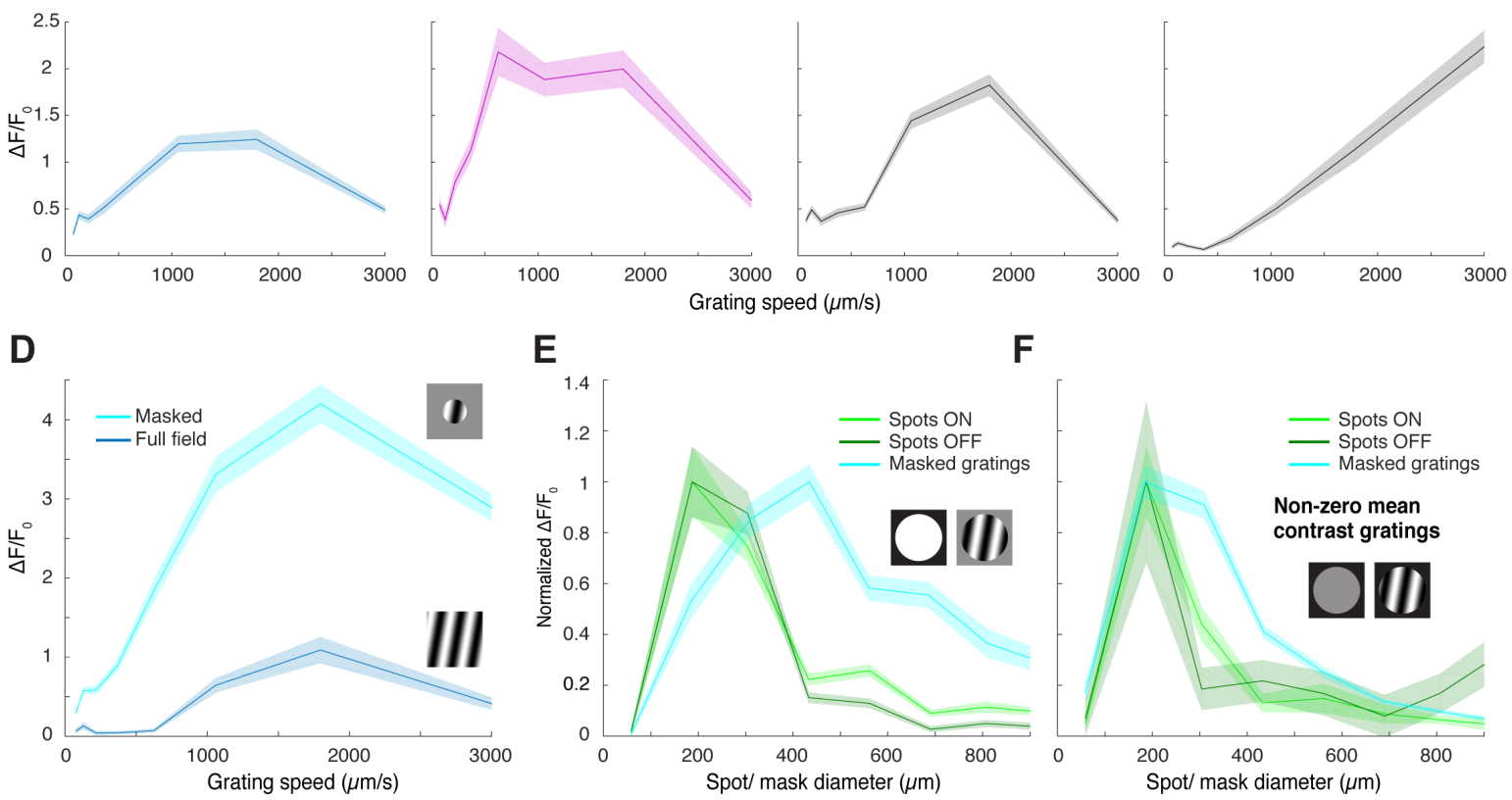

Figure 6. Calcium responses in VGluT3 AC dendrites. (A) Part of a field of view in the VGluT3 dendrite plexus, with ROIs (yellow) marked for movie analysis. (B) $\Delta \mathrm{F} / \mathrm{F}_{0}$ traces in two ROIs (top, bottom), in response to full-field gratings presented at different speeds. Individual trials, gray; Mean over trials, blue or magenta. Speeds of the grating are denoted above. (C) Response to full-field gratings vs. speed, averaged over ROIs, in four different FOVs. (D) Response vs. speed, averaged over ROIs, to full-field gratings vs. gratings inside a $230 \mu \mathrm{m}$ circular mask. (E) Response to bright spots vs. their diameter, appearing (ON, light green) or disappearing (OFF, dark green), presented from dark; Response to a drifting grating (Speed $1520 \mu \mathrm{m} / \mathrm{s}$, arbitrary angle, presented from gray), in a circular mask, vs. the mask diameter (Blue). (F) Same as in (E), but the spots and gratings contain an equal positive contrast relative to the background (both presented from dark, spots' contrast = gratings' contrast mean).

\section{Discussion}

The present work has shown that the mechanism underlying slow speed

tuning in ON DSGCs is feedforward inhibition suppressing firing at fast motion speeds, 
as has been found in rabbit. As in rabbit, this inhibition is primarily glycinergic, and electron microscopy data suggested that VGluT3 amacrine cells are the only likely source of glycine among amacrine cells that synapse onto ON DSGCs. The hypothesis that VGluT3 ACs provide the feedforward inhibition at high speeds required the existence of glycinergic synapses from VGluT3 to ON DSGCs that had not been previously found. Using optogenetics to specifically activate VGluT3 ACs and recording postsynaptic currents in ON DSGCs, we demonstrated the existence of these previously unknown functional synapses. By using chemogenetics to suppress neurotransmitter release from VGluT3 ACs and recording postsynaptic ON DSGCs, we have shown VGluT3 ACs contribute a major part of the inhibition that suppresses firing at high speeds. Finally, we have shown that VGluT3 ACs might affect the temporal tuning of cells in the retina beyond ON DSGCs by providing signal at high motion speeds (inhibition or excitation), and specifically of ON-OFF DSCGs.

The size of VGIUT3 ACs' relative contribution to inhibition in ON DSGCs at high speeds.

In the DREADD experiments with a drifting grating stimulus (Fig. 5A-E), application of CNO pushed down the speed-response curve by $\sim 40 \%$, which points to VGluT3 ACs account for at least $40 \%$ of this inhibition, most likely by direct transmission via the glycinergic synapses. When strychnine was subsequently applied, the maximum of the curve was reduced by $73 \%$ relative to the control curve, which suggests that at least $73 \%$ of the inhibition is mediated by glycine. Subsequent application of SR95531 then eliminated all inhibition, which implies that any inhibitory current left in the presence of CNO and strychnine is GABAergic. 
Lower bounds on VGluT3 and glycine contributions are stated above rather than exact values, as strychnine blocks glycine receptors everywhere in the retina, and might not only reduce the measured inhibitory current, but also increase it due to GABAglycine interactions upstream of the ON DSGC. The manipulation to block glycine release from VGluT3 using the DREADDs might be only partially effective.

What is the origin of glycine supposedly removed by strychnine but not CNO $(+23 \%$, above $)$ ? The DREADD experiment alone can not rule out a different source of glycine, such as a different type of amacrine cell. However, SBEM data suggested that VGluT3 is the only possibly source of glycine into ON DSGCs. This supports a different hypothesis, that the additional glycine is provided by VGluT3 ACs as well, but the DREADD system alone is only partially effective in blocking glycine release from them. A different piece of evidence also supported DREADDs being only partially effective, rather than the existence of another source of glycine. The effect of CNO application to suppress VGluT3 cells was a scaling down of the inhibitory currents, especially for high speeds. Beyond this the kinetics of the traces was preserved, and this suggests that the phenomenon seen before and after CNO application is the same, only attenuated, namely release from VGluT3. If the remaining current had come from a different type of amacrine cell, its kinetics may have been different.

Interestingly, strychnine applied in the DREADD experiment after CNO (Fig. $5 B$, reduced the currents more than strychnine applied alone in the same drifting grating experiment (Fig. 1D; 73\% vs. 57\% reduction). In the standard pharmacology experiment where only strychnine was applied, the peaks in the current traces following strychnine application were strongly out of phase with the peaks in the 
control traces, which suggested a GABA source that may have been artificially introduced with the application of strychnine (That, for example, could have different relative contributions of $\mathrm{ON}$ and $\mathrm{OFF}$ pathways, relative to the native inhibition) . In the DREADD experiment, with CNO and strychnine applied, not only was the speed response curve pushed down lower, but the kinetics of the remaining current was much more in phase with the kinetics in the control condition. This could point to an indirect GABA contribution from VGluT3 ACs, via glutamate exciting an intermediate GABAergic amacrine cell that in turn synapses on to the ON DSGC. A clue to the existence of the same pathway came from the optogenetics experiments, where in some ON DSGCs strychnine alone did not block all of the current induced by activation of VGluT3 ACs (Fig 4C), but glutamate receptor blockers did.

The evidence described above does not rule out some GABAergic contribution to the inhibition at high speeds. This could come from the other amacrine cells that have been observed in the SBEM data: SACs or wide filed amacrine cells. The experiments in rabbit[8] did not rule out a GABAergic contribution either, but rather suggested that glycine largely determined the tuning of ON DSGC responses to slow speeds. The results of standard pharmacology experiments in both species are similar. As in rabbit, we have been able to show a direct and dramatic effect of strychnine in increasing the spike responses of ON DSGCs at high speeds (Fig. 5F), which supports the major role of glycine. The effect of CNO alone on spiking was in the same direction (responses increase at high speeds) but was modest, possibly due to the partial effect of DREADDs discussed above. 
A novel functional glycinergic synapse between VGluT3 ACs and ON DSGCs.

SBEM data revealed synapses from VGluT3 ACs onto ON DSGCs, and guided by the search for a source of glycine in ON DSGCs, we have found previously unknown glycinergic synapses. Previous search for RGCs receiving excitation from VGluT3 ACs using optogenetics, found excitation in ON DSGCs[14], appearing as very transient peak of inward current of $\sim 150$ pA amplitude. We did not detect such excitation, although we rarely saw small and more sustained excitatory current, that was removed by blocking glutamate transmission. The difference could be due to our use of lower LED intensity ( $\sim 20$-fold). The same work found excitation but not inhibition from VGluT3 in ON-OFF DSGCs, findings that we replicated (Fig. 4F) (although this was not thoroughly tested, and excitation was more sustained in our observation). A following survey for targets of VGluT3 receiving inhibition[25] did not find inhibition in ON DSGCs, but found it in other RCGs (Suppressed by Contrast RGCs). This could conceivably be due to the use of a different VGluT3-cre mouse line that labels fewer of the VGluT3 ACs than in the present work. In an attempt we made in using a virus for cre-dependent expression of ChR2 (see methods) rather than the crossed mouse line, the expression seemed incomplete, and no postsynaptic currents were detected in ON DSGCs, although we were able to detect an inhibitory current in a Suppressed by Contrast RGC (Fig. 4I).

Implications for the optokinetic reflex.

Image stabilization on the retina is ubiquitous throughout seeing organisms, which implies that it is central to vision, presumably through increasing accuracy and 
speed and simplifying encoding. The OKR is a well preserved reflex among organisms from fish to primates. Here we have shown that the underlying mechanism for the speed tuning of the OKR is also similar, at least, between rabbit and mouse. The OKR relies both on information on direction and speed coming from the retinal DS system, as ON DSGC firing is limited to slow speeds. As for the directional component, the existence of SACs has been shown in humans[27,28], which suggested that direction selectivity and its underlying mechanism in the human retina are similar to that in rodents. As for motion speed, VGluT3 expressing amacrine cells have been found in baboon[29], with morphology and stratification that are similar to that in mouse. Thus the mechanism for speed tuning of the OKR could be conserved in humans as well, which in turn makes VGluT3 cells relevant for the human OKR and a potential target in future treatment of retinal pathologies. An important precedent exists for a component of the OKR mechanism found to play a similar role in mice and humans, the FRMD7 gene involved in establishing asymmetric SAC inhibition to horizontal motion preferring ON DSGCs in mouse[3].

Both in rabbit and in mouse, high speed motion triggers inhibition that suppresses or shuts down the response of ON DSGCs. Speed preference of excitatory inputs alone was not enough to account for the speed tuning of ON DSGCs. This is in contrast to a previous suggestion, that slow speed tuning in ON DSGCs arises as a result of a 'space-time wiring' arrangement of bipolar cells that evolved to enhance direction selectivity[18], an arrangement of slow and fast bipolar cell inputs of different types over the RGC dendrites. Direction selectivity of SAC processes is indeed believed to arise due such an arrangement[30,31]. While it may play a role at 
ON DSGC directions selectivity, an additional mechanism was needed to account for the slow speed tuning.

Thus slow speed tuning is not merely a corollary to the characteristics of the system, but rather a property that is tightly controlled using an additional layer of feedforward inhibition. There could be numerous reasons for why the output of ON DSGCs is actively shut down at high speeds, such as the need to prevent the OKR from counteracting fast types of eye movements like saccades[8] or minimization of energy expenditure under conditions for which signaling would be redundant due to the vestibular input.

High speed motion information from VGluT3 beyond ON DSGCs.

VGluT3 ACs now have many known target cell types in the retina[14,24,25,3234]. In one or more of these, VGluT3 ACs may signal fast speed motion and possibly shape speed tuning. The excitatory and inhibitory dual neurotransmitter property of them potentially enables them to affect speed sensitivity in opposite ways, by enhancing either inhibition or excitation at fast motion speeds. In the DS system, direction selectivity is if highly robust over a wide range of conditions, and that is in part due to the dual (excitatory and inhibitory) neurotransmitter property of SACs[35]. In contrast to ON DSGCs, ON-OFF DSGCs respond to motion up to fairly high speeds (Fig. 1C). We have found evidence for VGluT3 ACs increasing excitation and enhancing responses at high speeds, in agreement with the excitation found from VGluT3 to ON-OFF DS. Increasing the ON-OFF DS responses at high speeds might have an obvious benefit in detecting moving objects and directing gaze over a larger speed 
range. Our SBEM data also suggested that VGluT3 form synapses onto SACs, and our preliminary optogenetics data showed that these synapses are inhibitory. Past attempts to find excitation from VGluT3 in SACs detected none[14]. Further work will be needed to better understand the full effects VGluT3 ACs possibly have in the retinal DS system.

VGluT3 ACs might have an effect on fast speed motion sensitivity in RGCs outside of the DS system as well. OFF transient alpha cells, that receive excitation from VGluT3 are sensitive to motion up to high speeds (Fig. 1C). The ON delayed RGC is slow speed tuned. The response of this cell to a small spot of light is strongly delayed by early inhibition[16]. The ON delayed cell is one several RGCs that have been referred to as 'Suppressed by Contrast' or a 'Uniformity detector' [4,36]. It has a genetic marker[37], and receives VGluT3 inhibitory input[32], that may well be involved in suppressing its responses at high motion speed. A small ON-OFF bistratified RGC termed 'UHD' [4,38] was slow speed tuned in our sample. In addition, an RGC with similar morphology and stratification in rabbit, the local edge detector (LED), is tuned to low temporal frequency due to glycinergic inhibition suppressing its responses to fast changes in contrast[8,39]. In our preliminary optogenetics data, these cells receive inhibition from VGluT3 ACs. Previous work found that 'W3' ACs, that also refered to small bistartified RGCs, receive excitation form VGluT3 ACs $[14,24]$. This is, however, not in contradiction to the above, since RGCs that are bistratified between the ChAT bands include four different RGC subtypes[38]. Future studies may reveal how general the role of VGluT3 ACs is in signaling fast speed 
motion and fast contrast changes in the retina, and which RGCs and ACs make use of this signal.

\section{Methods}

Animals. All procedures were in accordance with the National Institutes of Health guidelines and approved by the Institutional Animal Care and Use Committee at Brown University. Detailed below are the strains of mice used, of adult mice of either sex, 2-8 months old. Wildtype C57BL/6J (Jackson Laboratory); To target ON DSGCs for recording, HoxD10-GFP (GENSAT collection, Tg(Hoxd10EGFP)LT174Gsat/Mmucd, MMRRC \#032065) and Pcdh9-cre (GENSAT collection, Tg(Pcdh9-cre)NP276Gsat/Mmucd, MMRRC \#036084) were used. The VGluT3-cre line (The Jackson Laboratory, B6;129S-Slc17a8 $8^{\text {tm1.1(cre)Hze } / J, ~ \# 028534) ~ w a s ~ c r o s s e d ~ w i t h ~}$ Ai32 (Jackson, B6.Cg-Gt(ROSA)26Sortm32(CAG-COP4*H134R/EYFP)Hze/J, \#024109) for optogenetics, (Jackson, B6.129-Gt(ROSA)26Sortm1(CAG-CHRM4*,-mCitrine)Ute/J, \#026219) for chemogenetics, and Ai14 (Jackson, B6;129S6-Gt(ROSA)26Sortm14(CAG-tdTomato)Hze/J, \#007908) for characterization of the VGluT3-cre mouse. For the Müller cell control experiment, (Jackson, Tg(Slc1a3-cre/ERT)1Nat/J, \#012586) was crossed with Ai32.

Retinal dissection. Isolation of the retina was performed similarly to [40]. The eyes were removed and immersed in oxygenated Ames medium (95\% 02, 5\% CO2; Sigma-Aldrich; supplemented with 23 mM NaHCO3 and 10 mM d-glucose). Under dim red light, the globe was cut, and cornea, lens and vitreous humour removed. A relieving ventral cut was made in the eyecup, and the retina was isolated. three more 
cuts were made in the retina, roughly along the temporal, nasal and dorso-nasal directions, the asymmetry of which was used to disambiguate retinal orientation. The retina was flat-mounted on a ploylysine coverslip (Corning, \#354086), which was secured in a recording chamber.

Tamoxifen injections. Tamoxifen (Sigma-Aldrich) was dissolved in corn oil (Sigma-Aldrich) to make $20 \mathrm{mg} / \mathrm{ml}$, sonicated (30 minutes, RT) and placed in hot water $\left(2 \mathrm{hrs}, 45^{\circ} \mathrm{C}\right)$ and once homogenous, filtered in a $0.2 \mu \mathrm{m}$ filter. Tamoxifen was injected IP, 2-2.5 mg per mouse, 3 days in a row. This resulted in dense YFP labeling of Müller glia in GLAST-cre x Ai32 mice.

Electrophysiology. Patch-clamp recordings of isolated flat-mount retina were performed under current-clamp using a Multiclamp 700B amplifier, Digidata 1550 digitizer, and pClamp 10.5 data acquisition software (Molecular Devices; $10 \mathrm{kHz}$ sampling). Pipettes were pulled from thick-walled borosilicate tubing (P-97, Sutter Instruments). Retinas were continuously superfused during experiments with oxygenated Ames' medium at $32{ }^{\circ} \mathrm{C}$, flow rate $\sim 5 \mathrm{ml} /$ minute. For cell attached recordings, Ames filled pipettes were used (tip resistance of 4-5 M $\Omega$ ). For whole cell voltage clamp recordings, pipettes filled with cesium internal solution (In mM: Cs methane sulfonate, 104.7, TEA-Cl, 10, HEPES, 20, EGTA, 10, QX-314, 2, ATP.Mg, 5, GTP-Tris, 0.5, pH 7.3, osmolarity 276 mOsm; All purchased from Sigma-Aldrich) were used (tip resistance of 5.5-6.5M $\Omega$ ). To isolate excitatory and inhibitory synaptic currents, the recorded cell was held near the reversal potential for inhibition $(\sim-65$ $\mathrm{mV}$ ) and excitation $(\sim+15 \mathrm{mV})$, respectively. Application of synaptic blockers to the bath as well as Clozapine-N-Oxide (CNO) was done by switching the perfused medium 
into medium containing the blocker and waiting for $\sim 7$ minutes. The blockers used were: strychnine (1 $\mu \mathrm{M}$, Sigma), SR95531 (10 $\mu \mathrm{M}$, Sigma), L-AP4 (20 $\mu \mathrm{M}$, Tocris),

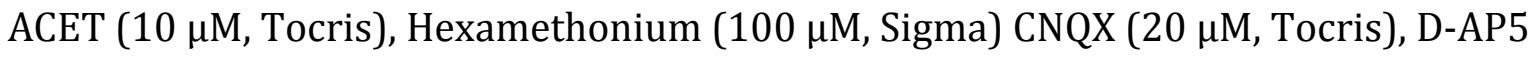
(50 $\mu \mathrm{M}$, Tocris). In the DREADD experiments, CNO was used to activate the DREADD (1 nM, Sigma).

Light stimulation. Light stimuli were generated as in [40]. Patterned visual stimuli, synthesized by custom software using Psychophysics Toolbox under Matlab (The MathWorks), were projected (AX325AA, HP) and focused onto the photoreceptor outer segments through the microscope's condenser. The projected display covered $\sim 1.5 \times 1.5 \mathrm{~mm}(5.8 \mu \mathrm{m} /$ pixel $)$. The video projector was modified to use a single UV LED lamp (NC4U134A, Nichia). The LED's peak wavelength (385 nm) shifted to $395 \mathrm{~nm}$ after transmission through a $440 \mathrm{~nm}$ short-pass dichroic filter (FF01-440/SP, Semrock), a dichroic mirror (T425lpxr, Chroma), and various reflective neutral density filters (Edmund Optics). The photoisomerization rates used were $10^{2}-10^{3} \mathrm{R}^{*} / \mathrm{rod} / \mathrm{s}$, and for the used spectrum were similar among rods, M-cones and S-cones. In the beginning of a stimulus sequence, a uniform screen with the stimulus' mean luminance was projected for 20-30s for light adaptation. To identify RGC types, the spike responses of the cell to a $230 \mu \mathrm{m}$ spot of +0.95 contrast were recorded. To assess the directional tuning of ON DSGCs we used a sinusoidal gratings $($ cycle $=380 \mu \mathrm{m}$, contrast $=0.95$, stimulus duration $=5$ s, interstimulus duration $=3 \mathrm{~s}$ at uniform mean grating luminance) drifted in 8 directions in a randomized sequence (drift speed $=230 \mu \mathrm{m} / \mathrm{s}, 4$ repetitions). For speed response 
curves, the same grating was drifted in the preferred direction, at 7-8 different speeds at a randomized sequence, with 3 repetitions for each speed.

Electrophysiology data analysis. Numbers with errors quoted in the text are mean \pm standard error of the mean, unless otherwise specified. All data analysis was done using custom written Matlab procedures. Individual peri-stimulus time histograms (PSTH) presented for spikes, or current traces for voltage clamp recordings, were averaged over three trials. Grey traces in Fig. 1B are individual trials. In population response vs. speed data, curves from different cells were normalized by their maximum and averaged. In population data where synaptic blockers or CNO were used (DREADDs), the response curves for each cell were normalized by the maximum of the control curve for that cell, and then curves were averaged over cells. The currents presented for optogenetics (Fig. 2) were recorded at $20 \mathrm{kHz}$, and in Fig. 2B-F,H-I current traces were averaged in 10ms windows. Current traces in Fig. 2 were averaged over 3-5 trials. Confocal and two-photon stacks were processed in Fiji (https://imagej.net/software/fiji), and projections were wither maximum intensity or maximum standard deviation projections.

Immunohistochemistry. Retinas were fixed and counterstained with the following antibodies: Goat anti-ChAT (Choline acetyltransferase; 1:200, Millipore Sigma \#AB144); Rabbit anti VGluT3 (1:250, Invitrogen \#PA5-85784). Chicken anti GFP (1:1000, Abcam \#ab13970) was used to enhance the fluorescence of the credependent GFP virus. Rabbit anti-HA tag (1: 200, Cell Signaling Technology \#3724) was used to stain the HA-tagged hM4Di receptor in the VGluT3 xR26 mouse. 
Imaging for cell targeting and dendritic morphology. To target fluorescent cells for patch recording, two photon imaging was used (Olympus FV1200MPE BASIC (BX61WI) microscope, 25× 1.05 NA water-immersion objective (XLPL25XWMP, Olympus), and an ultrafast pulsed laser (Mai Tai DeepSee HP, Spectra-Physics) tuned to $910 \mathrm{~nm}$ ). To acquire an image stack, RGCs were filled during electrophysiological recordings with Alexa hydrazide 488 or 594 (100 $\mu \mathrm{M}$, Invitrogen), and were imaged following the recording, either using the two-photon or the single-photon (confocal) configurations of the two-photon microscope. Tissue in which fluorescent proteins were expressed was often fixed and immunostained (see above), and subsequently imaged on a confocal microscope (Olympus FV3000, UPlan Super Apochromat objectives, 30xS, 1.05 NA, or 60x2S, 1.3 NA) in the Leduc Imaging Facility, Brown University.

Functional imaging. Imaging of calcium indicator signals were acquired using the aforementioned two-photon microscope and conditions, as has been done previously [40]. The frame rate was $15 \mathrm{~Hz}$. For imaging responses in dendrites, $128 \mathrm{x}$ 256 pixel fields of view were used with a zoom of 4.5-5x (60 $\mu \mathrm{m}$ x120 $\mu \mathrm{m}$ FOVs). Light stimulus presentation was synchronized to the fly-back times in the scanning of the microscope so that they did not interfere with the measured signal.

Functional imaging data analysis. Functional imaging analysis was done using Fiji and custom written Matlab routines. A maximum standard deviation projection of movies were made, over which ROIs were manually marked over brighter dendrites (Fig 6A), and traces of their area-averaged brightness over time were acquired. For the baseline fluorescence $\mathrm{F}_{0}$, we averaged the brightness over $0.5 \mathrm{~s}$ before every 
stimulus presentation. ROIs were chosen for analysis if their time averaged responses surpassed a threshold $\Delta \mathrm{F} / \mathrm{F}_{0}(0.3-0.6)$ during at least 6 stimulus presentations out of 24. Fig. 6B-F show curves averaged over responsive ROIs.

Intraocular injections. Mice were anaesthetized with isoflurane (3\% in oxygen; Matrx VIP 3000, Midmark). A viral vector inducing cre dependent expression of a payload (see below) was injected into the vitreous humour of the right eye through a glass pipette using a microinjector (Picospritzer III, Science Products GmbH). Analgesia (Proparicane, eye drops) was applied to the eye $\sim 2$ min before the injection, and immediately following the injection (Buprenorphine SR, $0.02 \mathrm{ml}$, intraperitoneal) to minimize postoperative pain. Mice were then taken off anesthesia, recovered within several minutes, and monitored for $48 \mathrm{hrs}$ following the procedure. Animals were killed and retinas removed 14-21 d later.

viruses. pAAV2/2-hSyn-DIO-hM4D(Gi)-mCherry (Addgene \#44362, Roth Lab) was injected intraocularly in VGluT3-cre mice, causing Cre-dependent expression of hM4D(Gi), a modified human muscarinic M4 receptor, that is an inhibitory Designer Receptor Exclusively Activated by Designer Drugs (DREADD). AAV2/2-EF1a-DIOhChR2(H134R)-EYFP (from UNC vector core, Deisseroth Lab) was injected VGluT3cre mice to express ChR2 in a cre dependent manner, but mostly caused expression in too few of the VGluT3 ACs to drive optogenetic responses in postsynaptic RGCs (Fig. 2E, Supp. Fig. 2D). rAAV2/2-CAG-flex-GFP (UNC vector core) was injected in Pcdh9cre mice to target ON DSGCs for recording.

Optogenetics. Light stimulation to activate ChR2 was generated using a LED light source (Mightex MLS-5500-MK1; LED driver: open-ephys.org, Cyclops) and 
introduced through the microscope objective and GFP excitation filter cube, resulting in a spectrum peak at $480 \pm 10 \mathrm{~nm}$, and illumination over an area of $1 \mathrm{~mm}$ in diameter. The light intensity at the sample was $0.9-1.4 \mathrm{nW} / \mu \mathrm{m}^{2}$, the lowest intensity that yielded robust postsynaptic responses in ON DSGCs. The stimulation time was $0.1 \mathrm{~s}$ or $1 \mathrm{~s}$ for 5 repeats, $4 \mathrm{~s}$ between repeats. The intensity and time were optimized for a robust response, while minimizing the rundown of the response over time, and the driving of large bursts of current that were sometimes observed, that were inconsistent over trials or not stimulus-locked.

Electron microscopy neuronal reconstructions. An existing dataset of retinal sections from a Serial Blockface Electron Microscope (SBEM), 'K0725’ [17] was used. The imaged volume dimensions were $50 \times 210 \times 260 \mu \mathrm{m}^{3}$ with the short dimension spanning the IPL and parts of the GCL and INL layers of the retina. The pixel size was $13.2 \mathrm{~nm}^{2}$ and the section thickness $26 \mathrm{~nm}$. The images contained intracellular details, e.g. synaptic vesicles. Tracing of dendritic skeletons and annotating synapses was done using the Knossos software package (https://webknossos.org).

\section{References}

1. Simpson, J.I. (1984). The Accessory Optic System. Annu. Rev. Neurosci. 7, 13-41.

2. Dhande, O.S., Estevez, M.E., Quattrochi, L.E., El-Danaf, R.N., Nguyen, P.L., Berson, D.M., and Huberman, A.D. (2013). Genetic dissection of retinal inputs to brainstem nuclei controlling image stabilization. J. Neurosci. 33, 17797-17813.

3. Yonehara, K., Fiscella, M., Drinnenberg, A., Esposti, F., Trenholm, S., Krol, J., 
Franke, F., Scherf, B.G., Kusnyerik, A., Müller, J., et al. (2016). Congenital Nystagmus Gene FRMD7 Is Necessary for Establishing a Neuronal Circuit Asymmetry for Direction Selectivity. Neuron 89, 177-193.

4. Goetz, J., Jessen, Z.F., Jacobi, A., Mani, A., Cooler, S., Greer, D., Kadri, S., Segal, J., Shekhar, K., Sanes, J., et al. (2021). Unified Classification of Mouse Retinal Ganglion Cells Using Function, Morphology, and Gene Expression. SSRN Electron. J., 1-27.

5. Sanes, J.R., and Masland, R.H. (2015). The Types of Retinal Ganglion Cells: Current Status and Implications for Neuronal Classification. Annu. Rev. Neurosci. 38, 221-246.

6. Bae, J.A., Mu, S., Kim, J.S., Turner, N.L., Tartavull, I., Kemnitz, N., Jordan, C.S., Norton, A.D., Silversmith, W.M., Prentki, R., et al. (2018). Digital Museum of Retinal Ganglion Cells with Dense Anatomy and Physiology. Cell 173, 12931306.e19.

7. Oyster, C.W., Takahashi, E., and Collewijn, H. (1972). Direction-selective retinal ganglion cells and control of optokinetic nystagmus in the rabbit. Vision Res. 12, 183-193.

8. Sivyer, B., Tomlinson, A., and Rowland Taylor, W. (2019). Simulated saccadic stimuli suppress ON-type direction-selective retinal ganglion cells via glycinergic inhibition. J. Neurosci. 39, 4312-4322.

9. Trenholm, S., Johnson, K., Li, X., Smith, R.G., and Awatramani, G.B. (2011). Parallel mechanisms encode direction in the retina. Neuron 71, 683-694. Available at: http://dx.doi.org/10.1016/j.neuron.2011.06.020. 
10. Kay, J.N., de la Huerta, I., Kim, I.J., Zhang, Y., Yamagata, M., Chu, M.W., Meister, M., and Sanes, J.R. (2011). Retinal ganglion cells with distinct directional preferences differ in molecular identity, structure, and central projections. J. Neurosci. 31, 7753-7762.

11. Grimes, W.N., Seal, R.P., Oesch, N., Edwards, R.H., and Diamond, J.S. (2011). Genetic targeting and physiological features of VGLUT3+ amacrine cells. Vis. Neurosci. 28, 381-392.

12. Haverkamp, S., and Wässle, H. (2004). Characterization of an Amacrine Cell Type of the Mammalian Retina Immunoreactive for Vesicular Glutamate Transporter 3. J. Comp. Neurol. 468, 251-263.

13. Johnson, J., Sherry, D.M., Liu, X., Fremeau, R.T., Seal, R.P., Edwards, R.H., and Copenhagen, D.R. (2004). Vesicular glutamate transporter 3 expression identifies glutamatergic amacrine cells in the rodent retina. J. Comp. Neurol. $477,386-398$.

14. Lee, S., Chen, L., Chen, M., Ye, M., Seal, R.P., and Zhou, J. (2014). An unconventional glutamatergic circuit in the retina formed by vGluT3 amacrine cells. Neuron $84,708-715$. Available at: http://dx.doi.org/10.1016/j.neuron.2014.10.021.

15. Kim, T., Soto, F., and Kerschensteiner, D. (2015). An excitatory amacrine cell detects object motion and provides feature-selective input to ganglion cells in the mouse retina. Elife 4,1-13.

16. Mani, A., and Schwartz, G.W. (2017). Circuit Mechanisms of a Retinal Ganglion Cell with Stimulus-Dependent Response Latency and Activation Beyond Its 
Dendrites. Curr. Biol. Available at:

http://dx.doi.org/10.1016/j.cub.2016.12.033.

17. Ding, H., Smith, R.G., Poleg-Polsky, A., Diamond, J.S., and Briggman, K.L. (2016).

Species-specific wiring for direction selectivity in the mammalian retina. Nature 535, 105-110. Available at: http://dx.doi.org/10.1038/nature18609.

18. Matsumoto, A., Briggman, K.L., and Yonehara, K. (2019). Spatiotemporally Asymmetric Excitation Supports Mammalian Retinal Motion Sensitivity. Curr. Biol. 29, 3277-3288.e5. Available at: https://doi.org/10.1016/j.cub.2019.08.048.

19. Zhang, C., and McCall, M.A. (2012). Receptor targets of amacrine cells. Vis. Neurosci. 29, 11-29.

20. Eggers, E.D., and Lukasiewicz, P.D. (2006). GABAA, GABAC and glycine receptormediated inhibition differentially affects light-evoked signalling from mouse retinal rod bipolar cells. J. Physiol. 572, 215-225.

21. Brüggen, B., Meyer, A., Boven, F., Weiler, R., and Dedek, K. (2015). Type 2 widefield amacrine cells in TH:: GFP mice show a homogenous synapse distribution and contact small ganglion cells. Eur. J. Neurosci. 41, 734-747.

22. Park, S.J.H., Borghuis, B.G., Rahmani, P., Zeng, Q., Kim, I.J., and Demb, J.B. (2015). Function and circuitry of VIP+ interneurons in the mouse retina. J. Neurosci. 35, 10685-10700.

23. Grimes, W.N., Aytürk, D.G., Hoon, M., Yoshimatsu, T., Gamlin, C., Carrera, D., Ahlquist, R.M., Sabnis, A., Diamond, J.S., Wong, R.O., et al. (2020). A high-density narrow-field inhibitory retinal interneuron with direct coupling to Müller glia. J. 
Neurosci., JN-RM-0199-20.

24. Krishnaswamy, A., Yamagata, M., Duan, X., Hong, Y.K., and Sanes, J.R. (2015).

Sidekick 2 directs formation of a retinal circuit that detects differential motion. Nature $524,466-470$.

25. Lee, S., Zhang, Y., Chen, M., and Zhou, Z.J. (2016). Segregated Glycine-Glutamate Co-transmission from vGluT3 Amacrine Cells to Contrast-Suppressed and Contrast-Enhanced Retinal Circuits. Neuron 90, 27-34. Available at: http://dx.doi.org/10.1016/j.neuron.2016.02.023.

26. Wienbar, S., and Schwartz, G.W. (2018). The dynamic receptive fields of retinal ganglion cells. Prog. Retin. Eye Res. 67, 102-117. Available at: https://doi.org/10.1016/j.preteyeres.2018.06.003.

27. Rodieck, R.W. (1989). Starburst amacrine cells of the primate retina. J. Comp. Neurol. 285, 18-37.

28. Yamada, E.S., Dmitrieva, N., Keyser, K.T., Lindstrom, J.M., Hersh, L.B., and Marshak, D.W. (2003). Synaptic connections of starburst amacrine cells and localization of acetylcholine receptors in primate retinas. J. Comp. Neurol. 461, 76-90.

29. Marshak, D.W., Chuang, A.Z., Dolino, D.M., Jacoby, R.A., Liu, W.S., Long, Y., Sherman, M.B., Suh, J.M., Vila, A., and Mills, S.L. (2015). Synaptic connections of amacrine cells containing vesicular glutamate transporter 3 in baboon retinas. Vis. Neurosci. 32.

30. Kim, J.S., Greene, M.J., Zlateski, A., Lee, K., Richardson, M., Turaga, S.C., Purcaro, M., Balkam, M., Robinson, A., Behabadi, B.F., et al. (2014). Space-time wiring 
specificity supports direction selectivity in the retina. Nature 509, 331-336.

Available at: http://dx.doi.org/10.1038/nature13240.

31. Fransen, J.W., and Borghuis, B.G. (2017). Temporally Diverse Excitation

Generates Direction-Selective Responses in ON- and OFF-Type Retinal Starburst Amacrine Cells. Cell Rep. 18, 1356-1365. Available at:

http://dx.doi.org/10.1016/j.celrep.2017.01.026.

32. Tien, N.W., Kim, T., and Kerschensteiner, D. (2016). Target-Specific Glycinergic Transmission from VGluT3-Expressing Amacrine Cells Shapes Suppressive Contrast Responses in the Retina. Cell Rep. 15, 1369-1375. Available at: http://dx.doi.org/10.1016/j.celrep.2016.04.025.

33. Jia, Y., Lee, S., Zhuo, Y., and Jimmy Zhou, Z. (2020). A retinal circuit for the suppressed-by-contrast receptive field of a polyaxonal amacrine cell. Proc. Natl. Acad. Sci. U. S. A. 117, 9577-9583.

34. Lee, S., Chen, M., Shi, Y., and Zhou, Z.J. (2021). Selective glycinergic input from vGluT3 amacrine cells confers a suppressed-by-contrast trigger feature in a subtype of M1 ipRGCs in the mouse retina. J. Physiol. 0, 1-14.

35. Sethuramanujam, S., McLaughlin, A.J., deRosenroll, G., Hoggarth, A., Schwab, D.J., and Awatramani, G.B. (2016). A Central Role for Mixed Acetylcholine/GABA Transmission in Direction Coding in the Retina. Neuron 90,1243-1256. Available at: http://dx.doi.org/10.1016/j.neuron.2016.04.041.

36. Jacoby, J., and Schwartz, G.W. (2018). Typology and circuitry of suppressed-bycontrast retinal Ganglion cells. Front. Cell. Neurosci. 12, 1-7.

37. Tien, N.W., Pearson, J.T., Heller, C.R., Demas, J., and Kerschensteiner, D. (2015). 
Genetically identified suppressed-by-contrast retinal ganglion cells reliably signal self-generated visual stimuli. J. Neurosci. 35, 10815-10820.

38. Jacoby, J., and Schwartz, G.W. (2017). Three small-receptive-field ganglion cells in the mouse retina are distinctly tuned to size, speed, and object motion. J. Neurosci. 37, 610-625.

39. Venkataramani, S., Van Wyk, M., Buldyrev, I., Sivyer, B., Vaney, D.I., and Taylor, W.R. (2014). Distinct roles for inhibition in spatial and temporal tuning of local edge detectors in the rabbit retina. PLoS One 9.

40. Sabbah, S., Gemmer, J.A., Bhatia-Lin, A., Manoff, G., Castro, G., Siegel, J.K., Jeffery, N., and Berson, D.M. (2017). A retinal code for motion along the gravitational and body axes. Nature 546, 492-497. Available at: http://dx.doi.org/10.1038/nature22818. 